\title{
Evaluación del impacto de la capacitación con lógica difusa
}

\author{
Impact assessment of training with fuzzy logic \\ Renier Esquivel García $\quad$ Gerardo Félix Benjamín ${ }^{1} \quad$ Rafael Bello Pérez ${ }^{2}$ \\ Recibido 28 de noviembre de 2012, aceptado 17 de junio de 2013 \\ Received: November 28, 2012 Accepted: June 17, 2013
}

\begin{abstract}
RESUMEN
Los recursos intangibles se han convertido en la principal fuente de generación de riqueza y bienestar dentro de la economía del conocimiento. El capital humano, como componente inherente a toda empresa, está conformado por un grupo de variables que agregan valor, por lo tanto, su medición es punto focal en las organizaciones del siglo XXI. Desafortunadamente, por el carácter intangible y calificativo de algunos de sus componentes, su medición y control presentan dificultades. El presente artículo propone, mediante el uso de herramientas basadas en la lógica difusa, evaluar el impacto de la capacitación en las empresas, aplicando el razonamiento propio de la lógica difusa a fin de complementar y extender la lógica clásica. El trabajo de ingeniería del conocimiento está basado en el conocimiento de los expertos, en el cual se identificaron los factores que incidían en el proceso, se modelaron estas como variables lingüísticas, se derivó un conjunto de reglas difusas para medir cualitativamente el impacto de la capacitación, se realizó una implementación de una aplicación en Matlab y se propuso un conjunto de casos al sistema con los que se obtuvieron muy buenos resultados.
\end{abstract}

Palabras clave: Lógica difusa, sistema de inferencia difusa, variables lingüísticas, capacitación, impacto de la capacitación.

\begin{abstract}
The intangible assets have become the main source of wealth creation and welfare within the knowledge economy. Human capital, as an inherent component in any company, is comprised of a group of variables that add value, therefore, its measurement is a focal point in the XXI century organizations. Unfortunately, due to the intangible and qualifying nature of some of its components, measurement and control of the human capital show some difficulties. This article proposes, through the use of tools based on fuzzy logic, the evaluation of the impact of training in companies, by applying the reasoning characteristic of fuzzy logic, with the aim to complement and extend the classical logic. The work of knowledge engineering is based on the knowledge of experts, in which the factors that influence on the process were identified and modeled as linguistic variables. A set of fuzzy rules was derived in order to measure qualitatively the impact of training. An implementation of an application in Matlab was developed and a set of cases was proposed to the system obtaining very good results.
\end{abstract}

Keywords: Fuzzy logic, fuzzy inference system, linguistic variables, training, impact of training.

\footnotetext{
1 Departamento de Capital Humano. Universidad de Sancti Spíritus "José Martí Pérez". Ave. de los Mártires \# 360. CP 60100, Sancti Spíritus, Cuba. E-mail: renier@uniss.edu.cu; gfelix@uniss.edu.cu

2 Departamento de estudios informáticos. Universidad Central "Marta Abreu" de Las Villas. Carretera de Camajuaní, kilómetro 8. Villa Clara, Cuba. E-mail: rbellop@uclv.edu.cu
} 


\section{INTRODUCCIÓN}

El entorno empresarial actual se caracteriza por el desarrollo de la información y la gestión del conocimiento. Para aprovechar esas oportunidades y minimizar las amenazas del mismo se debe entender que el principal valor de las organizaciones está en su capital intelectual y su gestión basada en el conocimiento y no solo en los activos tangibles, de ahí que la ventaja competitiva de las empresas en el siglo XXI radicará en el nivel de preparación de los recursos humanos [1].

Un aspecto importante dentro de la gestión del conocimiento es la correcta capacitación de todos los trabajadores, específicamente la de los dirigentes, debido a las exigencias del mundo globalizado actual, que ha hecho que las personas dentro de las organizaciones deban poseer diversas habilidades, las cuales les permitan desarrollar variadas actividades y adaptarse a distintas necesidades que el mismo entorno laboral y organizacional les exija. Como todo proceso, la capacitación de los dirigentes requiere ser evaluada sistemáticamente para valorar su efectividad, la evaluación involucra múltiples criterios, muchos de los cuales tienen un carácter subjetivo, resultando muy difícil realizar una medición exacta de los mismos. El carácter intangible de algunos de sus componentes, su medición y control presentan dificultades [2].

Por ello se puede utilizar la Teoría de los conjuntos difusos para desarrollar un procedimiento de evaluación más ajustado a la realidad. El enfoque que se propone permite tratar las dimensiones del impacto como variables linguiísticas y, basándose en ellas, formular criterios valorativos en forma de reglas difusas.

Con la aplicación de la lógica difusa al proceso de conocimiento se consigue una correspondencia más precisa con la realidad, se ofrece un nuevo modo de conocer la realidad, de construirla conceptualmente, con "operaciones lógicas que no responden a la estadística de la probabilidad ni por tanto a la frecuencia de un fenómeno, sino que construyen el razonamiento en términos de posibilidad, que son cualitativos con un vasto alcance epistemológico" y "en las ciencias humanas, no es más que reconocer la evidencia de que conceptos clave como los de cognición, emoción, inteligencia, mente, grupo, clase social, control social, opinión pública, institución social, son esencialmente difusos" [3].

La lógica difusa se ha comenzado a considerar como un elemento clave en el estudio de la realidad social, un contexto impregnado permanentemente de borrosidad como casi todo en las ciencias humanas. Algunas reflexiones críticas acerca de la lógica difusa y su aplicabilidad epistemológica a las ciencias sociales aparecen en [4], mientras que es vista como una nueva epistemología para las Ciencias de la Educación [5], la educación, por compleja, es caótica, es decir, incierta, y la lógica difusa es, paralelamente, una estrategia para abordar los problemas de incertidumbre. Incluso en las evaluaciones educativas -que pretenden afinar los niveles de certidumbre discriminando positiva o negativamente al alumno, a un centro o a un profesor por lo que aparecen los denominados cuantificadores difusos.

Debido a la creciente necesidad de darles una solución apropiada a esos problemas, que parten de percepciones estrictamente humanas y que como tal no cuentan con la suficiente información para aplicar modelos matemáticos convencionales, ha obligado a buscar modelos alternativos para llegar a valores numéricos a partir de variables expresadas en términos lingüísticos. Por ello la lógica difusa aparece como una de las herramientas que permiten hacer esa transformación y que proporciona una visión diferente a la otorgada por la lógica clásica [6]. Varios autores han abordado el tema sobre como evaluar el impacto de la capacitación, pero todos esos trabajos [7-18] evidencian acercamientos explicativos que no van mucho más allá que lo referido a los clásicos en el tema, lo que demuestra reproducción del conocimiento sin aportes científicos al tema. Cabe resaltar también como ejemplos de esas aproximaciones algunos intentos de explicar la adecuación metodológica de la lógica difusa a la educación. Un segundo grupo de trabajos está destinado a la evaluación de sistemas expertos y de sistemas tecnológicos de aprendizaje [19]. Trabajar en una solución al problema de la evaluación del impacto de la capacitación con técnicas basadas en conjuntos difusos resulta novedoso e interesante; además, los resultados alcanzados demuestran que es factible y permite crear modelos de evaluación eficaces. 
Un estudio técnico del problema permite encontrar que existen diferentes formas de representación del conocimiento, como las redes semánticas, los marcos, las ontologías y los mapas conceptuales [20] que permiten la exteriorización de conocimiento tácito y de construcción de conocimiento explícito, que combina la captura y la representación, de manera fácil y flexible, del conocimiento almacenado en la estructura cognitiva de la persona. Es importante señalar que la base de esa teoría es la espiral del conocimiento [21].

\section{SISTEMA DE INFERENCIA DIFUSA}

El hombre, en la búsqueda de la precisión, ha intentado ajustar el mundo real a modelos matemáticos rígidos y estáticos. El nacimiento de la teoría de los conjuntos difusos fue en la dirección contraria, generar modelos matemáticos adaptables a los problemas del mundo real; su desarrollo se debió a la necesidad de disponer de alguna representación matemática de familias de objetos usuales que, con la teoría clásica de conjuntos o la teoría de probabilidad no podían ser representados adecuadamente, ya que no son adecuadas para tratar la imprecisión, la incertidumbre, la no especificidad, la vaguedad, la inconsistencia y la complejidad del mundo real, permitiendo expresarlos en términos matemáticos capturando todas las incertidumbres asociadas con el razonamiento y el pensamiento humano.

La lógica difusa puede entenderse como una herramienta matemática cuya amplia aplicabilidad se basa en la concepción de conjuntos con fronteras no exactas que se emplean en presencia de información imperfecta [22-23], que se ocupa de los problemas relativos a la imprecisión, la incertidumbre y el razonamiento aproximado y es un marco que tolera la imprecisión y la verdad parcial bajo un enfoque no estadístico [24-25] y que puede ser construida basándose en el conocimiento de los expertos.

\section{Definición de conjuntos difusos}

La "teoría de conjuntos difusos" parte de la teoría clásica de conjuntos, añadiendo una función de pertenencia al conjunto $\mu$ generalmente definida como un número x entre 0 y 1 (el intervalo $[0,1]$ ) [26-29], en lugar de la pertenencia clásica binaria definida en el conjunto $\{0,1\}$. Así, se introduce el concepto de conjunto difuso asociado a un determinado valor lingüístico, definido por una palabra, adjetivo o etiqueta lingüística A. Entonces se puede decir que un conjunto difuso A se define como una función de pertenencia que enlaza o empareja los elementos de un dominio o universo de discurso $\mathrm{X}$ con elementos del intervalo [0,1], para cada conjunto difuso se define una función de pertenencia o inclusión $\mu_{\mathrm{A}}(\mathrm{x})$, que representa el grado en que un valor para la variable x está incluido en el concepto representado por la etiqueta A. Cuanto más cerca esté $\mathrm{A}(\mathrm{x})$ del valor 1 , mayor será la pertenencia del objeto $\mathrm{x}$ al conjunto $\mathrm{A}$. Los valores de pertenencia varían entre 0 (no pertenece en absoluto) y 1 (pertenencia total) por lo que un conjunto difuso es una clase de objetos con grados de pertenencias continuos. Existen varias definiciones de función de pertenencia [30-31]. Otros tipos de aproximación de números difusos son estudiados en los trabajos [32-35].

Otro concepto clave en la Teoría de los conjuntos difusos es el de variables lingüísticas, las mismas pueden formular descripciones vagas en lenguaje natural a términos matemáticos precisos y son una forma de comprimir información llamada granulación [36-37], ayudando a caracterizar fenómenos que están mal definidos o son complejos de definir o ambas cosas [38-40]. Formalmente una variable lingüística se define según [38-41] por $<\mathbf{N}, \mathbf{U}, \mathbf{T}(\mathbf{N}), \mathbf{M}>$ donde:

- $\quad \mathbf{N}$ es el nombre de la variable lingüística y $\mathbf{U}$ dominio subyacente.

- $\mathbf{T}(\mathbf{N})$ es el conjunto de términos o etiquetas lingüísticas que puede tomar $\mathbf{N}$.

- $\mathbf{M}$ es una regla semántica que asocia cada elemento de $\mathbf{T}(\mathbf{N})$ con un conjunto difuso en $\mathbf{U}$ de entre todos los posibles.

Las variables difusas proporcionan una transición gradual de estados y tienen capacidad para expresar y trabajar con observaciones y medidas de incertidumbre, que son más ajustadas a la realidad que las variables con cambios abruptos, de ahí que las etiquetas lingüísticas sean los términos lingüísticos definidos como conjuntos difusos que representan los posibles valores de una variable lingüística [42]. Utilizando las variables lingüísticas se pueden implementar los sistemas de inferencia difusa.

Un sistema de inferencia difusa es un sistema computacional basado en los conceptos de la teoría de conjuntos difusos, reglas IF-THEN difusas y 
razonamiento difuso. Su estructura básica consiste en tres componentes: una base de reglas difusas, una base de datos (la cual contiene las funciones de pertenencia usadas) y un mecanismo de razonamiento, que ejecuta el procedimiento de inferencia difusa; donde las entradas y salidas pueden ser valores duros o difusos. El mecanismo de inferencia difusa que se propone en el presente trabajo es de tipo Mamdani, por ser uno de los más empleados [43] y probados de manera práctica [44].

Cuando la salida es difusa y se necesita el valor duro, se emplea un método de defusificación que determina el valor duro que mejor representa el conjunto difuso. Para transformar esos resultados existen diversos métodos: el procedimiento máximo, el centro de gravedad, el centroide aproximado, el centro de área y operadores paramétricos [45-46].

\section{PROCEDIMIENTO PARA REALIZAR EL ANÁLISIS DIFUSO}

Para llevar a cabo un análisis difuso, lamentablemente no existe un procedimiento estándar, pero es posible definir ciertos pasos de trabajo para llegar a un buen diseño del sistema de inferencia difusa. La metodología que se propone para el presente trabajo utiliza el método de conocimiento experto, el cual sigue los lineamientos generales expuestos en [47-48] y que consta de cuatro componentes principales [4950]. Autores como [51-52] aplican esa metodología, que puede resumirse en los siguientes pasos:

1. Definir los objetivos, las restricciones y el comportamiento del sistema de inferencia difusa.

2. Selección de las variables.

3. Selección de la estrategia difusa o fusificación de las variables.

4. Construcción de la base de reglas.

5. Selección de la lógica de toma de decisiones, no es más que seleccionar el tipo específico de sistema de inferencia difusa.

6. Selección de la estrategia de defusificación.

7. Ejecutar pruebas para validar el sistema de inferencia difusa y verificar si la solución es compatible con el paso 1 . Se modifican los parámetros del sistema de inferencia difusa para aumentar la precisión del modelo.

7.1. Refinar el diseño.

7.2. Necesidad de mejora: Ir al paso 1.
El presente trabajo persigue evaluar el impacto de la capacitación con un modelo basado en un sistema de inferencia difusa que permita expresar las valoraciones sobre los indicadores en distintos dominios de expresión dependiendo de la naturaleza de los mismos o del conocimiento que tengan sobre ellos, que maneje la vaguedad o incertidumbre que esta conlleve. A continuación una breve descripción de las variables para realizar dicha evaluación, así como de sus respectivas etiquetas lingüísticas:

\section{Variables de entrada}

-Satisfacción del personal: expresa el grado de concordancia entre las expectativas que cada persona genera y las compensaciones que el curso le provee. -Satisfacción alta del personal: se observa una elevada participación y motivación por los cursistas, existen condiciones materiales y organizativas elevadas que contribuyen a un excelente criterio de los cursistas, así como excelente preparación de los profesores que enseñan con un elevado grado de aplicación en la práctica.

-Satisfacción media del personal: se manifiestan ausencias, y una participación media durante los cursos, existen ciertas deficiencias materiales y organizativas que pueden afectar la calidad de los cursos. Los contenidos no son del todo aplicables en la práctica del trabajo del dirigente.

-Satisfacción baja del personal: poca motivación con los cursos, falta de aplicación práctica para las funciones del dirigente, problemas materiales y organizativos.

-Aprendizaje: es el proceso por el que las personas adquieren conocimientos, técnicas y actitudes mediante la experiencia, la reflexión, el estudio o la instrucción, así como el resultado deseado tras la realización de un programa formativo.

-Aprendizaje alto: se manifiesta cuando los dirigentes adquieren los conocimientos y habilidades de forma satisfactoria, participando en todos los encuentros de forma activa y con elevada motivación.

-Aprendizaje medio: se evalúa el conocimiento y las habilidades de los dirigentes de manera positiva, observándose pequeñas deficiencias en el aprendizaje. Se adquieren algunos conocimientos y escasas habilidades.

-Aprendizaje bajo: se adquieren muy pocos conocimientos y habilidades, solo se cumplió con la presencia física del dirigente y no existe mucho interés por aprender. 
-Desempeño en el puesto: son las actividades que realiza el dirigente para cumplir con los objetivos establecidos. Cada empresa define los indicadores que considere para evaluar a sus dirigentes, los cuales pueden ser: cumplimiento de los objetivos de trabajo, responsabilidad, preocupación por sus subordinados, superación, intercambio con todos los trabajadores de la empresa, entre otros.

-Desempeño elevado: el dirigente evidencia elevados resultados en su trabajo, excelentes criterios de sus subordinados y superiores, logra los objetivos establecidos con el máximo aprovechamiento de los cursos recibidos.

-Desempeño medio: cuando se realiza un trabajo satisfactorio en el puesto de trabajo cumpliendo con todo lo establecido. Los criterios de sus subordinados y superiores son buenos y los indicadores de desempeño que forman parte del mismo se cumplen con algunos señalamientos.

-Desempeño bajo: existe un mal trabajo, lo que pone de manifiesto un bajo rendimiento en la mayoría de los indicadores del desempeño que posee la empresa, mostrándose reiterados incumplimientos de los objetivos establecidos por la institución.

-Clima organizacional: es la forma en que el dirigente percibe su trabajo, su rendimiento, su productividad y satisfacción en la labor que desempeña. Se evalúa de acuerdo con el liderazgo, comunicación, trabajo en equipo, condiciones de trabajo, estimulación, motivación y nivel de capacitación.

-Clima organizacional favorable: se observa un favorable ambiente de trabajo y excelente relación entre los factores del sistema organizacional de la empresa y el comportamiento individual tiene consecuencias positivas sobre la organización.

-Clima organizacional medio: se observan insatisfacciones y un ambiente de trabajo, que no se cumple en toda su totalidad. Además, los trabajadores manifiestan inconformidades en alguno de los indicadores que integran el clima organizacional.

-Clima organizacional desfavorable: existen quejas y un desfavorable ambiente de trabajo, lo que implica consecuencias negativas sobre la organización. Los trabajadores manifiestan criterios negativos en factores como liderazgo, comunicación, trabajo en equipo, condiciones de trabajo, estimulación, motivación y nivel de capacitación.

-Disciplina laboral: cumplimiento de los objetivos del puesto de trabajo en correspondencia con los objetivos de la empresa, lo cual exige tiempo de trabajo dedicado a los mismos.

-Disciplina laboral buena: se refleja en el cumplimiento de los objetivos del puesto de trabajo en correspondencia con los objetivos de la empresa. No se manifiestan dificultades en el ausentismo, impuntualidad, desaprovechamiento de la jornada, incumplimiento de las normas de trabajo, incumplimiento de la calidad del trabajo, desobediencia a la administración, entre otras.

-Disciplina laboral regular: se cumple parcialmente con los objetivos establecidos en el puesto de trabajo, incurriendo en algunas pérdidas de tiempo imputables al comportamiento de los empleados, afectando los objetivos que se desean alcanzar. Los índices que miden una buena disciplina laboral no se satisfacen en su totalidad.

Disciplina laboral mala: incumplimiento de los objetivos del puesto de trabajo en correspondencia con los objetivos de la empresa, pérdidas de tiempo imputables al comportamiento de los empleados. Aparecen incumplimientos en casi todos los índices de la misma.

-Resultados económicos: es el cumplimiento de los objetivos de la empresa en términos económicos, reflejando los beneficios y pérdidas de la misma. Es el aporte social de la institución.

-Resultados económicos buenos: se cumplen los indicadores económicos de la empresa, evidenciándose un elevado aporte social, se sobrecumplen los planes y se observa satisfacción en los empleados.

-Resultado económico regular: existen algunos señalamientos por los auditores, superiores y trabajadores, no se cumple el plan en toda su totalidad. -Resultados económicos bajos: no se cumple con los indicadores económicos establecidos en el período, no se cumple el plan.

\section{Variables de salida}

-Impacto individual: es la repercusión que tiene la capacitación recibida, mediante cambios favorables en el nivel de satisfacción, competencias de los individuos y su desempeño en el puesto de trabajo, evaluándose en alto, medio o sin impacto.

-Impacto organizacional: es la repercusión que tiene la capacitación recibida, mediante cambios favorables en el mejoramiento de la eficacia y la eficiencia, evaluándose en alto, medio o sin impacto. 


\section{Modelamiento del problema}

Para dar solución al problema se diseñó un sistema de inferencia difusa, en el cual cada una de las variables anteriores fue modelada como una variable lingüística. Como las valoraciones lingüísticas son estimaciones que pueden obtenerse mediante la consulta a expertos, se permitió que para cada etiqueta lingüística fueran asignados valores numéricos en el intervalo $[0,10]$, representadas adecuadamente con números difusos triangulares y trapeciales. Se escogió una escala comprendida entre 0 y 10 $([0,10])$ para identificar el universo de discurso de todas las variables lingüísticas.

A partir de los criterios anteriores, dentro del universo de discurso de cada variable lingüística se asignó un conjunto de etiquetas lingüísticas que aparecen en la Tabla 1, donde se describe el intervalo difuso donde los valores de las etiquetas lingüísticas poseen $100 \%$ de pertenencia al conjunto difuso al cual pertenecen. Por solo mencionar un ejemplo, la variable satisfacción del personal consta de las etiquetas lingüísticas "satisfecho", "algo" e "insatisfecho", en el que la etiqueta lingüística "satisfecho" representa el nivel más alto que puede alcanzar dicha variable, la etiqueta lingüística algo, el nivel medio y así respectivamente, todo en correspondencia con las descripciones antes mencionadas. Ello significa que la variable satisfacción del personal en su etiqueta lingüística satisfecho tenga un grado de pertenencia de 0 cuando alcanza el valor de 5, de $1(100 \%)$ cuando la variable obtenga el valor comprendido de 9 a 10, lo mismo sucede en el intervalo de 0 a 1 para la etiqueta lingüística "insatisfecho". Ello da una medida de que cuando el valor de la variable se va incrementando a partir de 5 hasta alcanzar el valor de 9 , el grado de pertenencia también aumentará hasta alcanzar 1 (100\%); lo contrario sucede del valor 9 al 5 .

\section{Construcción de las funciones de pertenencia}

Para la construcción de las funciones de pertenencia se adquirió la información de encuestas aplicadas a expertos, donde se tomaron los valores de pertenencia según el número de respuestas favorables. Dada la subjetividad y ambigüedad de las opiniones de los expertos, las funciones de pertenencia construidas se corrigieron de acuerdo con la comparación de las opiniones de los especialistas con las respuestas esperadas, así como a los estudios de comportamiento de dichas variables lingüísticas. Ello se logró para cada indicador en particular.
En la presente investigación, los conjuntos difusos que representan las variables relativas que intervien en la evaluación del impacto de la capacitación se asumen de la siguiente forma: $\mathrm{y}=\{\mu(\mathrm{x}) \mid \mathrm{x}\}$, donde $\mu(x)$ denota el grado de pertenencia de $x$, definiendo $x$ como valor del universo de cada etiqueta lingüística. Para mejor comprensión remitirse a la Figura 1 y ver la Tabla 2, donde se observa la representación de la variable lingüística satisfacción del personal a modo de ejemplo.

Tabla 1. Variables y Etiquetas lingüísticas.

\begin{tabular}{|c|c|c|c|}
\cline { 2 - 4 } \multicolumn{1}{c|}{} & \multicolumn{3}{c|}{ INTERVALO DIFUSO } \\
\cline { 2 - 4 } \multicolumn{1}{c|}{} & {$[9,10]$} & 5 & {$[0,1]$} \\
\hline $\begin{array}{c}\text { VARIABLES } \\
\text { LINGÜÍSTICAS }\end{array}$ & \multicolumn{2}{c|}{ ETIQUETAS LINGÜÍSTICAS } \\
\hline $\begin{array}{c}\text { Satisfacción del } \\
\text { personal }\end{array}$ & Satisfecho & Algo & Insatisfecho \\
\hline Aprendizaje & Mucho & Medio & Poco \\
\hline $\begin{array}{c}\text { Desempeño } \\
\text { en el puesto }\end{array}$ & Elevado & Señalamiento & Bajo \\
\hline $\begin{array}{c}\text { Clima } \\
\text { Organizacional }\end{array}$ & Favorable & $\begin{array}{c}\text { Menos } \\
\text { favorable }\end{array}$ & Desfavorable \\
\hline Disciplina laboral & Buena & Regular & Mala \\
\hline $\begin{array}{c}\text { Resultados } \\
\text { económicos }\end{array}$ & Buen & Regular & Mal \\
\hline Impacto individual & Alto & Medio & Bajo \\
\hline $\begin{array}{c}\text { Impacto } \\
\text { organizacional }\end{array}$ & Alto & Medio & Bajo \\
\hline $\begin{array}{c}\text { Impacto de la } \\
\text { capacitación }\end{array}$ & Alto & Medio & Bajo \\
\hline
\end{tabular}

Fuente: Elaboración propia.

Tabla 2. Representación explícita de la función de pertenencia difusa de la variable satisfacción del personal.

\begin{tabular}{|c|c|c|c|}
\hline \multicolumn{4}{|c|}{ FUNCIONES DE PERTENENCIA DIFUSAS } \\
\hline Variables & \multicolumn{3}{|c|}{ Etiquetas lingüísticas } \\
\hline \multirow{2}{*}{$\begin{array}{l}\text { Satisfacción } \\
\text { del personal }\end{array}$} & Satisfecho & Algo & Insatisfecho \\
\cline { 2 - 4 } & $\{0 \mid 51191110\}$ & $\{0|2,51150| 7,5\}$ & $\left\{\begin{array}{llll}110 & 111 & 0 \mid 5\end{array}\right\}$ \\
\hline
\end{tabular}

Fuente: Elaboración propia.

Gráficamente todas las variables lingüísticas tienen el comportamiento que se muestra en la Figura 1.

Relaciones entre las variables y la variable objetivo La combinación de las variables y su calificación mediante indicadores permite medir el estado o nivel en que se encuentra el impacto individual y el impacto organizacional y, por ende, el impacto de 


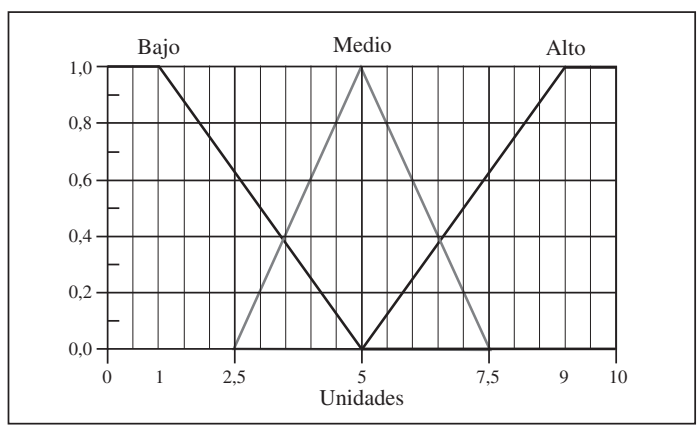

Figura 1. Conjunto difuso de la variable de entrada y salida del sistema de inferencia difusa. Fuente: Elaboración propia.

la capacitación. La unión de las dos dimensiones: el impacto a nivel individual y el impacto organizacional pasarán a integrar el conjunto de entrada para evaluar el impacto de la capacitación.

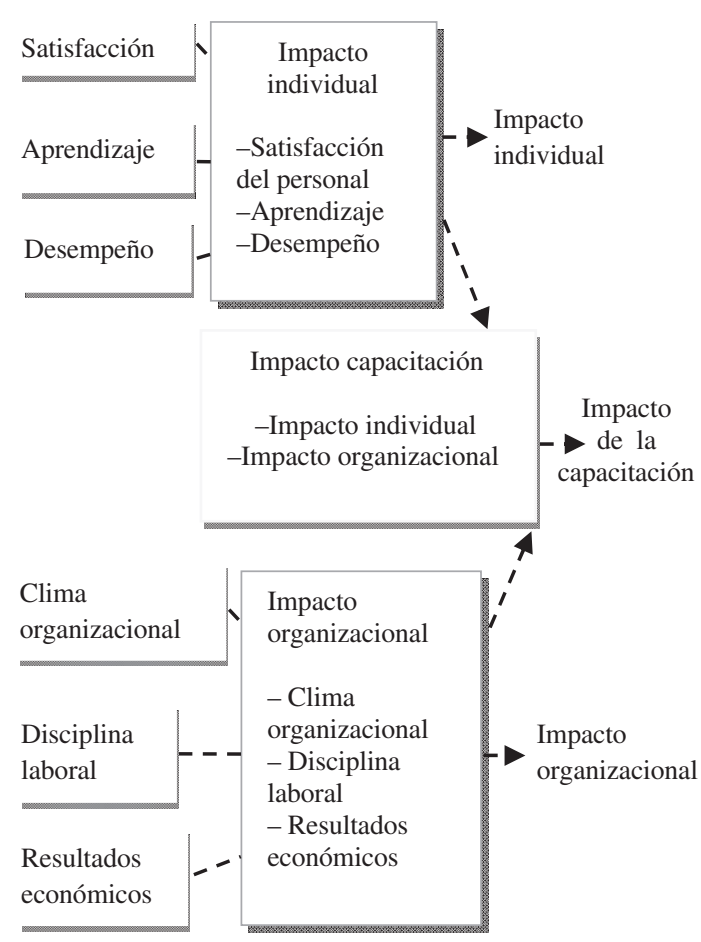

Figura 2. Estructura del sistema de inferencia difusa.

Fuente: Elaboración propia.

En la Figura 2 se muestra la estructura del sistema de inferencia difusa. Los conjuntos difusos y los operadores difusos forman el sujeto y verbo de la lógica difusa y la forma de comprimirlos es mediante las reglas difusas. Las reglas difusas son la representación en lenguaje natural del conocimiento (empírico o no) que se tiene acerca de un problema del mundo real. Para la construcción de las reglas difusas se utilizaron las relaciones causales descritas en la Figura 2. A continuación se muestra la Tabla 3 y se describe de la forma SI-ENTONCES las relaciones antes mencionadas en un sencillo ejemplo.

Ello demuestra que si el personal está satisfecho con el curso, aprende mucho y demuestra un elevado desempeño laboral entonces hay un impacto a nivel individual alto. Si hay un clima organizacional favorable, buena disciplina laboral y buen resultado económico en la organización entonces el impacto a nivel organizacional también es alto. La obtención de un impacto individual y organizacional alto permite un alto impacto en la capacitación.

Tabla 3. Sistema de inferencia difusa.

\begin{tabular}{|l|l|l|l|}
\hline \multicolumn{2}{|c|}{ SÍ } & \multicolumn{2}{c|}{ ENTONCES } \\
\hline $\begin{array}{l}\text { Satisfacción } \\
\text { del personal }\end{array}$ & Aprendizaje & $\begin{array}{l}\text { Desempeño } \\
\text { en el puesto }\end{array}$ & \multicolumn{1}{|c|}{$\begin{array}{c}\text { Impacto } \\
\text { individual }\end{array}$} \\
\hline Satisfecho & Mucho & Elevado & Alto \\
\hline $\begin{array}{l}\text { Clima } \\
\text { organizacional }\end{array}$ & $\begin{array}{l}\text { Disciplina } \\
\text { laboral }\end{array}$ & $\begin{array}{l}\text { Resultados } \\
\text { económicos }\end{array}$ & $\begin{array}{l}\text { Impacto } \\
\text { organizacional }\end{array}$ \\
\hline Favorable & Buena & Buen & Alto \\
\hline \multicolumn{2}{|l|}{ Impacto de la capacitación } & Alto \\
\hline
\end{tabular}

Fuente: Elaboración propia.

\section{Evaluación de la solución propuesta}

Para validar la eficacia del sistema de inferencia difusa construido para evaluar el impacto de la capacitación, se consulta un panel de especialistas constituido por un total de siete, con experiencia y conocimientos del tema de investigación, lo que garantiza la confiabilidad de los resultados.

Para llegar a un acuerdo grupal de las opiniones se aplicó el método experto (Delphi por rondas) o criterio de expertos propuesto en [53] para respaldar dichas afirmaciones. El criterio de selección fue tomado para los casos donde existe una concordancia de 7 a 0,6 a 1 y 5 a 2 respectivamente, es decir, para las situaciones donde exista una concordancia mayor o igual a $60 \%$. Las reglas finales quedaron determinadas por las características de ese proceso y además fueron configuradas para cumplir con normas elementales como: 
- COMPLETITUD: Las reglas han de cumplir todas las combinaciones posibles de entradas al controlador, con el fin de que no queden fisuras en las cuales no se tome una acción.

- CONSISTENCIA: No pueden coexistir dos acciones de control para la misma situación, lo que produciría una contradicción.

- INTERACCIÓN: Corresponde al peso propio que tiene una regla entre las restantes, para ello se debe analizar el efecto de las reglas en la acción de control.

- ROBUSTEZ: Es una medida de la reacción del controlador frente a perturbaciones en las entradas.

Una parte de la base de conocimiento por la que está conformado el sistema de inferencia difusa está dada como reglas difusas según muestra la Tabla 4.

Tabla 4. Base datos de conocimiento para el impacto individual.

\begin{tabular}{|c|c|c|c|}
\hline \multicolumn{3}{|c|}{ BASE DE CONOCIMIENTO } \\
\hline \multicolumn{3}{|c|}{ IMPACTO INDIVIDUAL } \\
\hline \multicolumn{2}{|c|}{ Sí } & \multicolumn{2}{c|}{ ENTONCES } \\
\hline $\begin{array}{c}\text { Satisfacción } \\
\text { del personal }\end{array}$ & Aprendizaje & $\begin{array}{c}\text { Desempeño } \\
\text { en el puesto }\end{array}$ & $\begin{array}{c}\text { Impacto } \\
\text { individual }\end{array}$ \\
\hline Satisfecho & Mucho & Elevado & Alto \\
\hline Algo & Mucho & Elevado & Alto \\
\hline Insatisfecho & Mucho & Elevado & Medio \\
\hline Satisfecho & Medio & Elevado & Medio \\
\hline Algo & Medio & Elevado & Medio \\
\hline Insatisfecho & Medio & Elevado & Medio \\
\hline Satisfecho & Poco & Elevado & Alto \\
\hline Algo & Poco & Elevado & Medio \\
\hline Insatisfecho & Poco & Elevado & Sin impacto \\
\hline Satisfecho & Mucho & Señalamiento & Alto \\
\hline Algo & Mucho & Señalamiento & Alto \\
\hline Insatisfecho & Mucho & Señalamiento & Medio \\
\hline
\end{tabular}

Fuente: Elaboración propia.

Luego de haber analizado las variables lingüísticas con sus respectivas combinaciones mediante reglas difusas se utilizó el método de defusificación centro de gravedad o centroide.

\section{Valoración experimental}

Para dar una valoración experimental del sistema de inferencia difusa fueron propuestos y formulados más de 100 casos, otorgándoles valores a todas las variables de entrada y realizando un análisis con el resultado de salida dado por el software con los otorgados por el panel de expertos. Comparando los datos analizados se observa que el experimento arrojó una alta coincidencia de los valores de salida con los criterios generados por los especialistas. Para validar la robustez con que el sistema responde a las distintas interrogantes a partir de los datos de entrada, se hizo uso del programa estadístico informático Statistical Package for the Social Sciences (SPSS), el cual es muy usado en las ciencias sociales [54].

En la Tabla 5 se observan las variables de salida del sistema, junto con los resultados de haber aplicado el test de Friedman, las curvas ROC (que no es más que la certeza con que cada regla es evaluada según corresponda, o sea, bajo, medio y alto) y las tablas de contingencia.

Para la variable Impacto individual, a pesar de que se aprecian diferencias significativas entre los expertos (significación 0,002 con intervalo de confianza a la izquierda de 0,05 , e incluso de 0,01 , después de haberse aplicado el test de Friedman); puede notarse en la Tabla 6, que cuando se relacionan los resultados del sistema con la tendencia central de los siete expertos, se obtienen resultados satisfactorios. Así queda evidenciado con las tres curvas ROC, las cuales tienen área significativamente mayor que 0,5 . Además se alcanzan buenos resultados con el uso de la Tabla 6 de contingencia para dicha variable, por lo que no existen discordantes extremos (Alto-Bajo).

Para la variable Impacto organizacional después de aplicar el test de Friedman se observa que tampoco hay diferencias significativas según la Tabla 5, lo cual indica concordancia entre los expertos. Las 3 curvas ROC que se hacen con la certeza del sistema propuesto respecto de las correspondientes evaluaciones de los expertos 1,2,3 se aprecia una

Tabla 5. Indicadores de validación en el SPSS.

\begin{tabular}{|l|c|c|c|c|c|}
\hline Variables & $\begin{array}{c}\text { Sig. de } \\
\text { Friedman }\end{array}$ & $\begin{array}{c}\text { Curva } \\
\text { ROC } \\
\text { Bajo }\end{array}$ & $\begin{array}{c}\text { Curva } \\
\text { ROC } \\
\text { Medio }\end{array}$ & $\begin{array}{c}\text { Curva } \\
\text { ROC } \\
\text { Alto }\end{array}$ & $\begin{array}{c}\% \text { de } \\
\text { casos } \\
\text { concor- } \\
\text { dantes }\end{array}$ \\
\hline $\begin{array}{l}\text { Impacto } \\
\text { individual }\end{array}$ & 0,002 & 0,859 & 0,809 & 0,827 & 76,7 \\
\hline $\begin{array}{l}\text { Impacto } \\
\text { organizacional }\end{array}$ & 0,339 & 0,942 & 0,813 & 0,887 & 71,7 \\
\hline $\begin{array}{l}\text { Impacto } \\
\text { capacitación }\end{array}$ & 0,023 & 0,919 & 0,821 & 0,910 & 80,0 \\
\hline
\end{tabular}

Fuente: Elaboración propia. 
Tabla 6. Tabla de contingencia entre los expertos y el sistema para la variable Impacto individual.

\begin{tabular}{|c|c|c|c|c|c|c|}
\hline \multicolumn{7}{|c|}{ Expertos*Sistema } \\
\hline & & & \multicolumn{3}{|c|}{ Sistema } & \multirow{2}{*}{ Total } \\
\hline & & & Bajo & Medio & Alto & \\
\hline \multirow{3}{*}{$\begin{array}{c}\text { Exper- } \\
\text { tos }\end{array}$} & Bajo & $\begin{array}{l}\text { Frecuencia } \\
\% \text { de los } \\
\text { expertos }\end{array}$ & $\begin{array}{c}4 \\
100\end{array}$ & & & $\begin{array}{c}4 \\
100\end{array}$ \\
\hline & Medio & $\begin{array}{l}\text { Frecuencia } \\
\% \text { de los } \\
\text { expertos }\end{array}$ & $\begin{array}{c}1 \\
2,9\end{array}$ & $\begin{array}{c}28 \\
82,4\end{array}$ & $\begin{array}{c}5 \\
14,7\end{array}$ & $\begin{array}{c}34 \\
100\end{array}$ \\
\hline & Alto & $\begin{array}{l}\text { Frecuencia } \\
\% \text { de los } \\
\text { expertos }\end{array}$ & & $\begin{array}{c}8 \\
36,4\end{array}$ & $\begin{array}{c}14 \\
63,6\end{array}$ & $\begin{array}{c}22 \\
100\end{array}$ \\
\hline \multicolumn{2}{|c|}{ Total } & $\begin{array}{l}\text { Frecuencia } \\
\% \text { de los } \\
\text { expertos }\end{array}$ & $\begin{array}{c}5 \\
8,3\end{array}$ & $\begin{array}{c}36 \\
60,0\end{array}$ & $\begin{array}{c}19 \\
31,7\end{array}$ & $\begin{array}{c}60 \\
100\end{array}$ \\
\hline
\end{tabular}

Sig. de Monte Carlo para el test exacto de Fisher $=0,000$. La concordancia exacta entre el sistema y los expertos se logra en un 46 de los 60 casos para un 76,6\%.

Fuente: Elaboración propia con el SPSS.

Tabla 7. Tabla de contingencia entre los expertos y el sistema para la variable Impacto organizacional.

\begin{tabular}{|c|c|c|c|c|c|c|}
\hline \multicolumn{7}{|c|}{ Expertos*Sistema } \\
\hline & & & \multicolumn{3}{|c|}{ Sistema } & \multirow{2}{*}{ Total } \\
\hline & & & Bajo & Medio & Alto & \\
\hline \multirow{3}{*}{ Expertos } & Bajo & $\begin{array}{l}\text { Frecuencia } \\
\% \text { de los } \\
\text { expertos }\end{array}$ & $\begin{array}{c}4 \\
80,0\end{array}$ & $\begin{array}{c}1 \\
20,0\end{array}$ & & $\begin{array}{c}5 \\
100\end{array}$ \\
\hline & Medio & $\begin{array}{l}\text { Frecuencia } \\
\% \text { de los } \\
\text { expertos }\end{array}$ & $\begin{array}{c}4 \\
13,8\end{array}$ & $\begin{array}{c}21 \\
72,4\end{array}$ & $\begin{array}{c}4 \\
13,8\end{array}$ & $\begin{array}{c}29 \\
100\end{array}$ \\
\hline & Alto & $\begin{array}{l}\text { Frecuencia } \\
\% \text { de los } \\
\text { expertos }\end{array}$ & & $\begin{array}{c}8 \\
30,8\end{array}$ & $\begin{array}{c}18 \\
69,2\end{array}$ & $\begin{array}{c}26 \\
100\end{array}$ \\
\hline \multicolumn{2}{|c|}{ Total } & $\begin{array}{l}\text { Frecuencia } \\
\% \text { de los } \\
\text { expertos }\end{array}$ & $\begin{array}{c}8 \\
13,3\end{array}$ & $\begin{array}{c}30 \\
50,0\end{array}$ & $\begin{array}{c}22 \\
36,7\end{array}$ & $\begin{array}{c}60 \\
100\end{array}$ \\
\hline
\end{tabular}

Sig. de Monte Carlo para el test exacto de Fisher $=0,000$. La concordancia exacta entre el sistema y los expertos se logra en un 43 de los 60 casos para un 71,6\%.

Fuente: Elaboración propia con el SPSS.

buena concordancia (véase que todas las áreas bajo las curvas ROC tienen valores superiores a $0,8 \mathrm{e}$ intervalos de confianza francamente a la derecha de 0,5 , que es el área de la recta diagonal del cuadrado que indicaría mala clasificación). Por último, la Tabla 7 de contingencia muestra que no existen discordantes extremos (Alto-Bajo).
En la variable Impacto de la capacitación, según la Tabla 5, se observan diferencias significativas entre los expertos. No obstante, cuando se relacionan los resultados del sistema con la tendencia central de los siete expertos, se obtienen resultados satisfactorios. Así queda evidenciado con las tres curvas ROC, todas las cuales tienen área significativamente mayor que 0,5 . También queda demostrado con la Tabla 8 .

\section{CONCLUSIONES}

La lógica difusa tiene una historia corta, pero un rápido crecimiento debido a su capacidad de resolver problemas relacionados con la incertidumbre de la información o del conocimiento de los expertos. Además, proporciona un método formal para la expresión del conocimiento en forma entendible por todos. En el trabajo presentado en el artículo se ha utilizado la misma para crear un modelo computacional que permite evaluar el impacto de la capacitación en una institución dada. Se creó un sistema inteligente, definido como sistema de inferencia difusa, que permite evaluar el impacto de la capacitación y constituye una herramienta que permite tomar decisiones en la definición de acciones para mejorar la capacitación empresarial. La valoración experimental mediante el empleo de un conjunto de casos de prueba mostró un desempeño satisfactorio del sistema. Tiene la ventaja de utilizar

Tabla 8. Tabla de contingencia entre los expertos y el sistema para la variable Impacto de la capacitación.

\begin{tabular}{|c|c|c|c|c|c|c|}
\hline \multicolumn{7}{|c|}{ Expertos*Sistema } \\
\hline & & & \multicolumn{3}{|c|}{ Sistema } & \multirow{2}{*}{ Total } \\
\hline & & & Bajo & Medio & Alto & \\
\hline \multirow{3}{*}{ Expertos } & Bajo & $\begin{array}{l}\text { Frecuencia } \\
\% \text { de los } \\
\text { expertos }\end{array}$ & $\begin{array}{c}7 \\
70,0\end{array}$ & $\begin{array}{c}3 \\
30,0\end{array}$ & & $\begin{array}{c}10 \\
100\end{array}$ \\
\hline & Medio & $\begin{array}{l}\text { Frecuencia } \\
\% \text { de los } \\
\text { expertos }\end{array}$ & $\begin{array}{c}1 \\
3,1\end{array}$ & $\begin{array}{c}24 \\
75,0\end{array}$ & $\begin{array}{c}7 \\
21,9\end{array}$ & $\begin{array}{c}32 \\
100\end{array}$ \\
\hline & Alto & $\begin{array}{l}\text { Frecuencia } \\
\% \text { de los } \\
\text { expertos }\end{array}$ & & $\begin{array}{c}1 \\
5,6\end{array}$ & $\begin{array}{c}17 \\
94,4\end{array}$ & $\begin{array}{c}18 \\
100\end{array}$ \\
\hline \multicolumn{2}{|c|}{ Total } & $\begin{array}{l}\text { Frecuencia } \\
\% \text { de los } \\
\text { expertos }\end{array}$ & $\begin{array}{c}8 \\
13,3\end{array}$ & $\begin{array}{c}28 \\
46,7\end{array}$ & $\begin{array}{c}24 \\
40,0\end{array}$ & $\begin{array}{c}60 \\
100\end{array}$ \\
\hline
\end{tabular}

Sig. de Monte Carlo para el test exacto de Fisher $=0,000$. La concordancia exacta entre el sistema y los expertos se logra en un 48 de los 60 casos para un $80 \%$.

Fuente: Elaboración propia con el SPSS. 
un sistema inteligente y agilizar el tiempo de trabajo de los evaluadores en las áreas de capacitación.

\section{REFERENCIAS}

[1] A. Cuesta. "Tecnología de Gestión de Recursos Humanos". Félix Varela y Academia. Cuarta Edición. La Habana, Cuba. Vol. 1, pp. 347. 2010. ISBN: 978-959-07-1340-8 OC.

[2] F. Benjamín y R. Esquivel. "Procedimiento para evaluar el impacto de la capacitación en los cuadros de las empresas que contribuyen al desarrollo local utilizando Lógica Difusa". Tesis para optar al grado de licenciado. Universidad Central "Marta Abreu" de Las Villas. Villa Clara, Cuba. 2011.

[3] F. Munné. "Las teorías de la complejidad y sus implicaciones en las ciencias del comportamiento". Revista Interamericana de Psicología. Vol. 29 No 1, pp. 1-12.1995. ISSN: 0034-9690.

[4] J. Calventus. "La lógica borrosa como aporte a una nueva epistemología en ciencias sociales: una aproximación conceptual". Revista Psicología. Vol. $1 \mathrm{~N}^{\mathrm{o}}$ 2, pp. 1-18. 2000. ISSN: 0719-0581.

[5] L. Ballester y A. Colom. "Lógica difusa: una nueva epistemología para las Ciencias de la Educación”. Revista de Educación. Vol. 340, pp. 995-1008. 2006. ISSN: 0034-8082.

[6] S. Medina. "Aproximación a la medición del capital intelectual organizacional aplicando sistema de inferencia difusa". Cuadernos de Administración. Vol. 23, pp. 35-68. 2010. ISSN: 1900-7205.

[7] M. Agüero. "Evaluación del impacto de la capacitación de la Maestría en Dirección de un grupo de directivos empresariales de Ciudad de La Habana". I Taller científico nacional sobre la medición del impacto de la capacitación. Matanzas, Cuba. 2003.

[8] Y. Castro y R. Esquivel. "Diseño e implementación de un procedimiento para la evaluación del impacto de la capacitación recibida por la reserva de cuadros de la UJC de Sancti Spíritus". Tesis para optar al grado de Máster en Dirección. Universidad de Sancti Spíritus "José Martí Pérez". Sancti Spíritus, Cuba. 2007.

[9] Z. Barreto y R. Esquivel. "Diseño e implementación de un procedimiento de formación y desarrollo que permita conocer el impacto de la capacitación en la gerencia territorial de ETECSA". Tesis para optar al grado de licenciada en Contabilidad y Finanzas. Universidad de Sancti Spíritus "José Martí Pérez”. Sancti Spíritus, Cuba. 2007.

[10] J. Cabrera. "Repensar la evaluación del impacto de la capacitación". Impacto de la capacitación. Editado por Dirección de Capacitación de Cuadros y Estudios de Dirección. Primera Edición. La Habana, Cuba. Vol. 1, pp. 6-25. 2004. ISBN: 959-16-0274-8.

[11] M. Clemente y R. Esquivel. "Evaluación del impacto de la capacitación en la granja agroindustrial Aracelio Iglesias". Tesis para optar al grado de licenciada en Contabilidad y Finanzas. Universidad de Sancti Spíritus "José Martí Pérez”. Sancti Spíritus, Cuba. 2007.

[12] J. Diez y J. Abreu. "Impacto de la capacitación interna en la productividad y estandarización de procesos productivos: un estudio de caso". Daena: International Journal of Good Conscience. Vol. 4 NN $^{\circ}$ 2, pp. 97-144. 2009. ISSN: ISSN 1870-557X.

[13] R. Esquivel y E. Gutiérrez. "Implementación de un procedimiento que facilite la medición del impacto de la capacitación de los cuadros y sus reservas en el CAI Arrocero "Sur del Jíbaro". Tesis para optar por el grado de Máster en Dirección. Universidad de Sancti Spíritus “José Martí Pérez". Sancti Spíritus, Cuba. 2006.

[14] H. Garza. "Impacto de la capacitación en una empresa del ramo eléctrico". Daena: International Journal of Good Conscience. Vol. $4 \mathrm{~N}^{\circ}$ 2, pp 194-249. 2009. ISSN: ISSN 1870-557X.

[15] Y. López y R. Esquivel. "Evaluación del impacto de la capacitación en el CAI Arrocero "Sur del Jíbaro" para mejorar la Gestión Empresarial". Tesis para optar por el grado de Máster en Dirección. Universidad de Sancti Spíritus "José Martí Pérez". Sancti Spíritus, Cuba. 2007.

[16] S. Pérez. "Propuesta Metodológica para la Evaluación de Impacto de la Maestría en Dirección impartida por la Facultad de Ciencias Económicas y Empresariales de la Universidad de Oriente". II Taller Nacional de Medición del Impacto de la Capacitación. Matanzas, Cuba. 2004. 
[17] F. Rabaza. "La medición del impacto de la capacitación: El caso de un Diplomado". I evento sobre la Evaluación de Impacto de la capacitación. Matanzas, Cuba, 2003.

[18] L. Rodríguez y R. Esquivel. "Procedimiento para evaluar el impacto de la capacitación en los cuadros y sus reservas en las organizaciones que favorecen el desarrollo local del municipio de Yaguajay". Tesis para optar al grado de licenciada en Cultura Física. Universidad de Sancti Spíritus "José Martí Pérez”. Sancti Spíritus, Cuba. 2010.

[19] G. Feixas y J. Cornejo. "Manual de la técnica de la rejilla mediante el programa RECORD ver. 2.0". Paidós. Segunda edición. Barcelona, España. Vol. 1, pp. 95. 1996. ISBN: 84-493-0317-6.

[20] A. Simón y V. Estrada. "Herramientas para el perfeccionamiento de los sistemas de gestión de conocimiento basados en mapas conceptuales". Tesis para optar al grado de doctor. Instituto Superior Politécnico "José Antonio Echeverría”. La Habana, Cuba. 2008.

[21] I. Nonaka y H. Takeuchi. "The Knowledge Creating Company: How Japanese Companies Create the Dynamics for Innovation". Oxford University Press. First edition. New YorkOxford, USA. Vol. 1, pp. 1-95. 1995. ISBN: 0-19-509269-4.

[22] A. Kandel. "Fuzzy Expert Systems". CRC Press. Primera edición. Florida, USA. Vol. 1, pp. 99-112. 1992. ISBN: 084934297X.

[23] L. Zadeh. "Fuzzy sets, fuzzy logic, and fuzzy systems: selected papers". World Scientific Pub. Co. Inc. Singapore. Vol. 6, pp. 821. 1996. ISBN: 9810224214.

[24] L.Zadeh. "Nacimiento y evolución de la lógica borrosa, el soft computing y la computación con palabras: un punto de vista personal". Psicothema. Vol. 8 No 2, pp. 421-429. 1996. ISSN: 0214-9915.

[25] C. Carlsson and R. Fuller. "Fuzzy reasoning in decision making and optimization". PhysicaVerlag Heidelberg. New York, USA. Vol. 82, pp. 337.2002. ISBN: 3-7908-1428-8.

[26] J. Jang. "Neuro fuzzy and soft computing: A computation al approach to learning and machine intelligence". Prentice Hall. New York, USA. Vol. 1, p. 614. 1997. ISBN: 0132610663.
[27] A. Kulkarni. "Computer vision and fuzzyneuronal systems". Prentice Hall PTR. New York, USA. Vol. 1, p. 509. 2001. ISBN: 0135705991.

[28] N. Kasabov. "Foundations of neural networks, fuzzy systems and knowledge engineering". The MIT Press. Segunda edición. Massachusetts, USA. Vol. 1, p. 547. 1998. ISBN: 0-262-11212-4.

[29] B. Kosko. "El futuro borroso o el cielo en un chip". Drakontos Bolsillo. Primera edición. Barcelona, España. Vol. 1, pp. 25-35. 2010. ISBN: 978-84-9892-087-1.

[30] B. Kosko. "Neural Networks and Fuzzy Systems: A Dynamical Systems Approach to Machine Intelligence". Prentice Hall. New York, USA. Vol. 1, p. 449. 1992. ISBN: 0136114350.

[31] J. Galindo. "Conjuntos y sistemas difusos. Lógica difusa y apliaciones". E.T.S.I. Informática. 2007. Fecha de consulta: 2012. URL: http://www.lcc.uma.es/ ppgg/FSS/

[32] Y. Chalco-Cano, M. Jiménez-Gamero, H. Román-Flores and M.A. Rojas-Medar. "An approximation to the extension principle using decomposition of fuzzy intervals". Fuzzy Sets and Systems. Vol. 159, Issue 24, pp. 3245-3258. 2008. ISSN: 0165-0114.

[33] G. González-Rodríguez, A. Colubi and W. Trutschnig. "Simulation of fuzzy random variables". Information Sciences. Vol. 179, Issue 5, pp. 642-653. 2009. ISSN: 0020-0255.

[34] E. Nasibov and S. Peker. "On the nearest parametric approximation of a fuzzy number". Fuzzy Sets and Systems. Vol. 159, Issue 11, pp. 1365-1375. 2008. ISSN: 0165-0114.

[35] L. Stefanini, L.Sorini and M.L. Gerra. "Parametric representation of fuzzy numbers and application to fuzzy calculus". Fuzzy Sets and Systems. Vol. 157, Issue 24, pp. 2423-2455. 2006. ISSN: 0165-0114.

[36] L. Zadeh. "Soft Computing and Fuzzy Logic". IEEE Software. Vol. 11, Issue 6, pp. 48-56. 1994. ISSN: 0740-7459.

[37] L. Zadeh. "Fuzzy Logic, Neural Networks, and Soft Computing. The concept of a linguistic variable and its application to approximate reasoning-II. The concept of a linguistic variable and its application to approximate reasoning-III". Commun. ACM. Vol. 37, Issue 3, pp. 77-84. 1994. ISSN: 0001-0782. 
[38] L. Zadeh. "The concept of a linguistic variable and its application to approximate reasoning-III". Information Sciences. Vol. 9, pp. 43-80. 1975. ISSN: 0020-0255.

[39] L. Zadeh. "The concept of a linguistic variable and its application to approximate reasoning-I". Information Sciences. Vol. 8, Issue 3, pp. 199-249. 1975. ISSN: 0020-0255.

[40] L. Zadeh. "The concept of a linguistic variable and its application to approximate reasoning-II". Information Sciences. Vol. 8, pp. 301-375. 1975. ISSN: 0020-0255.

[41] L. Zadeh. "Outline of a new approach to analysis of complex systems and decision processes". IEEE Trans. syst. Man and Cybernetics. Vol. 3, Issue 1, pp. 22-44. 1973. ISSN: 0018-9472.

[42] V. Soler. "Lógica difusa aplicada a conjuntos imbalanceados: Aplicación a la detección del síndrome de Down". Tesis para optar al grado de doctor. Universitat Autònoma de Barcelona. Barcelona, España. 2007.

[43] E.H. Mamdani and S. Assilian. "An Experiment in Linguistic Synthesis with a Fuzzy Logic Controller”. International Journal of Man-Machine Studies. Vol. 7, Issue 1, pp. 1-13. 1975. ISSN: 0020-7373.

[44] E. Mamdani. "Application of Fuzzy Logic to Approximate Reasoning Using Linguistic Synthesis". IEEE Trans. Computers. Vol. 26, Issue 12, pp. 1182-1191. 1977. ISSN: 0018-9340.

[45] H. Bandemer and S. Gottwald. "Fuzzy sets, fuzzy logic, fuzzy methods with applications". John Wiley and Sons. Primera edición. New York, USA. Vol. 1, p. 250. 1995. ISBN: 0471956368.
[46] G. Tejada. "Tutorial de lógica Fuzzy". Electrónica-UNMSM. Vol 5, pp. 18-29. 2000.

[47] C. Lee. "Fuzzy Logic in Control Systems: Fuzzy Logic Controllers-II". IEEE Trans. on Systems, Man and Cybernetics. Vol. 20, pp. 419-433. 1990. ISSN: 0018-9472.

[48] C. Lee. "Fuzzy Logic in Control Systems: Fuzzy Logic Controllers-I". IEEE Trans. on Systems, Man and Cybernetics. Vol. 20, Issue 2, pp. 404-435. 1990. ISSN: 0018-9472.

[49] W. Pedrycz. "Fuzzy Control and Fuzzy Systems". Research Studies Press. Second edition. Taunton, Somerset, England. Vol. 1, p. 350. 1993. ISBN: 0863801315.

[50] R. Yager and D. Filev. "Essentials of Fuzzy Modeling and Control". Wiley Interscience. New York, USA. Vol. 1, p. 388. 1994. ISBN: 0471017612.

[51] C. Martínez. "Uso de las Técnicas de Preprocesamiento de Datos e Inteligencia Artificial (Lógica Difusa) en la Clasificación/ Predicción del Riesgo Bancario". Tesis para optar al grado de Licenciado. Universidad de los Andes. 2007.

[52] L. Augier. "Tecnología para evaluar el desempeño de los recursos humanos en entidades turísticas". TURyDES. Vol. 3 No 8. 2010. ISSN: 1988-5261.

[53] L. Cabrera y R. Esquivel. "Procedimiento para identificar las competencias laborales en la Universidad de Sancti Spíritus José Martí Pérez”. Tesis para optar al grado de ingeniero industrial. Universidad de Sancti Spíritus "José Martí Pérez”. Sancti Spíritus, Cuba. 2010.

[54] A. Pardo y M. Ruiz. "SPSS 11. Guía para el análisis de datos". McGraw-Hill. Primera Edición. España. Vol. 1, p. 736. 2002. ISBN: 978-84-481-3750-2. 